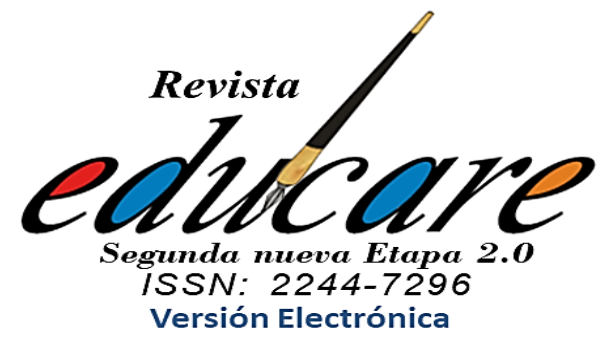

Volumen 25 No. 2 Mayo - Agosto 2021

$(215-236)$

\title{
EL MODELO FLIPPED CLASSROOM EN LA EDUCACIÓN VIRTUAL: UNA EXPERIENCIA EN MATEMÁTICAS UNIVERSITARIAS
}

ORCIID: https://orcid.org/0000-0002-5216-3069

Universidad Politécnica Salesiana (Ecuador)

\author{
THE MODEL FLIPPED CLASSROOM IN \\ VIRTUAL EDUCATION: AN EXPERIENCE IN \\ UNIVERSITY MATHEMATICS
}




\section{Introducción y Estado de la Cuestión}

Desde el inicio de la pandemia del año 2020 causada por el COVID-19 la educación tradicional se ha visto forzada a cambiar drásticamente de un entorno presencial a otro virtual (Engelbrecht et al. 2020). Actualmente, las clases virtuales se han convertido en la cotidianidad de cada estudiante a todos los niveles educativos y en todo el mundo (CEPAL-UNESCO, 2020). Tomando en cuenta que hasta entonces el uso de la tecnología era bastante marginado en la educación (Cevikbas y Kaiser, 2020), los docentes se vieron obligados a instruirse con un ritmo muy acelerado en las nuevas tecnologías de la información y comunicación (TIC) utilizadas en el ambiente educativo.

Como consecuencia, la calidad educativa se ha visto comprometida (Amaya, Cantú y Marreros, 2021), pues, no hay garantía de que los logros de aprendizaje lleguen de "manera virtual" a los estudiantes. Un indicador que lo evidencia es la poca participación que tienen los estudiantes en sus procesos de aprendizaje (Bagley, 2020), como posible consecuencia del estrés, sobrecarga académica y/o frustración llevando a una eventual deserción (Lovón y Cisneros, 2020).

Por ello, es una necesidad motivar de alguna manera la participación de los alumnos en clase (Ramirez, 2020). Para atacar esta situación, desde hace varios años muchos docentes ya han estado innovando en sus prácticas educativas utilizando diversos métodos de enseñanza ajustados a las herramientas tecnológicas con las que se cuenta (Blasco-Serrano et al., 2016; Castellanos et al., 2017; Latorre-Cosculluela et al., 2020; Matzumura-Kasano et al., 2018).

En particular, enseñar matemáticas siempre ha sido un desafío que tienen los docentes de esta asignatura a causa de la complejidad y abstracción de la misma (Lucas y Gascón, 2019). En una modalidad virtual, buscar métodos de enseñanza que ataquen estos desafíos se ha vuelto uno de los principales enfoques de muchos investigadores de didáctica de las matemáticas que buscan responder al enigmático escenario que envuelve los procesos de enseñanza-aprendizaje de la matemática en ambientes virtuales (Aldon y Panero, 2020; Borba et al. 2016; Cevikbas y Kaiser, 2020; De Araujo et al. 2017; Ndlovu et al. 2020; Trouche et al. 2020; Vahey et al. 2020; Voigt et al. 2020).

Basados en lo anterior, el problema de investigación de este estudio estuvo enfocado en la poca participación de los estudiantes en sus procesos de aprendizaje durante las clases virtuales de matemática, considerándose el método de enseñanza utilizado como una de las 
principales causas de la problemática. Por ello, se propuso aplicar el método Flipped Classroom (FC) en dos asignaturas de matemáticas universitarias (álgebra lineal y cálculo diferencial) que fueron enseñadas de manera virtual. Así pues, el objetivo de esta investigación fue determinar la eficacia del modelo FC aplicado en las clases virtuales de matemática sobre la participación de los estudiantes en sus procesos de aprendizaje. A tal fin, se planteó la siguiente pregunta de investigación:

¿Cómo el modelo flipped classroom usado en las clases virtuales de matemática puede aumentar la participación del estudiante en su proceso de aprendizaje?

\section{Concepto de Flipped Classroom}

El modelo pedagógico flipped classroom (FC), o de acuerdo a su traducción del inglés, aula invertida, como su nombre lo indica consiste en "invertir" las situaciones didácticas tradicionalmente tratadas en el aula (Sánchez-Rivas et al., 2019). La intención es que los estudiantes inviertan tiempo fuera de clase trabajando individualmente para adquirir los conocimientos básicos del contenido del curso (Mcgivney-Burelle y Xue, 2013). Para Martínez-Olvera y Esquivel-Gámez (2018) este modelo consiste en voltear los roles y espacios tradicionales de enseñanza, de manera que los conceptos que generalmente son enseñados en el aula sean atendidos por los estudiantes antes de la clase y las actividades prácticas sean tratadas en ella.

En concordancia, Bagley (2020) define el FC como un enfoque de instrucción que intercambia las actividades en clase con las asignadas fuera de ella, es decir, una parte del contenido que normalmente es presentado en el aula se asigna previamente al estudiante (generalmente por medio de videos); liberando tiempo de la clase para implementar metodologías activas y dar un seguimiento y apoyo puntual a las necesidades de cada estudiante (Martínez-Olvera y Esquivel-Gámez, 2018).

Por su parte, un grupo de docentes investigadores que forman parte de la comunidad Flipped Learning Network (FLN) han desarrollado una definición que va más allá de invertir el aula, pues consideran que realmente lo que debe invertirse es el aprendizaje. De acuerdo a esto el aprendizaje invertido lo definen como un enfoque pedagógico en el que la instrucción directa se mueve desde el espacio de aprendizaje grupal al espacio de aprendizaje individual. En consecuencia, el espacio grupal resultante se transforma en un entorno de aprendizaje dinámico e interactivo donde el docente guía a los estudiantes a medida que aplican conceptos 
y se involucran creativamente en el tema en cuestión (Flipped Learning Network, 2014). En este estudio se entendió que el FC necesariamente debía implicar un aprendizaje invertido. De acuerdo con FLN (2014), apoyada luego por investigaciones más recientes (Basso-Aránguiz et al. 2018; Blasco-Serrano et al. 2016; Cevikbas y Kaiser, 2020), el docente debe incorporar en su metodología de aula invertida los siguientes cuatro pilares fundamentales:

Un ambiente flexible donde el estudiante pueda elegir cuándo y dónde estudiar, para ello es necesario que el profesor diseñe espacios de aprendizajes apoyados en la tecnología que permitan flexibilizar los plazos de aprendizaje y evaluaciones de los estudiantes.

Una cultura de aprendizaje basada en una pedagogía que centra su atención en el estudiante, quien siendo el responsable de efectuar una revisión previa de los contenidos a tratar puede dedicar el tiempo de clase a explorar temas de mayor complejidad participando activamente en la construcción de su conocimiento. Infundir esta cultura de aprendizaje es uno de los principales retos que tiene el docente desde el inicio del proceso.

Un contenido intencional que debe ser organizado y categorizado por el docente, es decir, el profesor debe decidir cuáles serán los contenidos que el estudiante podrá manejar de manera autónoma por medio de los recursos audiovisuales propuestos, y cuáles deberán ser orientados y profundizados por él en la clase presencial. Este contenido debe estar enmarcado dentro del programa analítico de la asignatura.

Un docente profesional que termina siendo el pilar más importante de esta metodología, quien a pesar de tener el papel menos visible en un aula invertida termina siendo el responsable de garantizar el éxito de sus estudiantes, debe observar continuamente la evolución de ellos y brindarles una retroalimentación oportuna. Además, debe ser reflexivo en su práctica docente y capaz de aceptar críticas constructivas para mejorar su instrucción en beneficio de su alumnado.

Hasta el momento, el FC se ha abordado de distintas maneras, sin embargo, todas convergen con que es una pedagogía centrada en el estudiante. Donde el docente debe diseñar actividades de aprendizaje en ambientes virtuales que faciliten el aprendizaje diferenciado (Cevikbas y Kaiser, 2020). Al mismo tiempo que aborden los conocimientos básicos relacionados con definiciones, habilidades y procedimientos de rutina mediante lecturas, videos u otros recursos en línea (Martínez-Olvera y Esquivel-Gámez, 2018), entendiendo que los estudiantes necesitan menos apoyo al momento de desarrollar este tipo de actividades (Mcgivney-Burelle y Xue, 2013); además, los autores recalcan la importancia de hacer evaluaciones breves antes de asistir a clase y asegurar un fundamento teórico-conceptual que 
permita un punto de partida para iniciar con actividades que involucren un razonamiento de nivel superior.

En vista de esto, la tecnología juega un papel fundamental en la aplicación de este modelo pedagógico y su incorporación debe promover un ambiente donde el estudiante sea el principal responsable de su aprendizaje guiado por el docente con una evaluación sistemática y una retroalimentación adecuada (Basso-Aránguiz et al., 2018). A tal fin, los estudiantes deben recibir oportunamente materiales multimedia diseñados y compartidos en algún sistema de gestión de aprendizaje (Moodle, Blackboard, Schoology, Google Classroom, entre otros); favoreciendo el aprendizaje autónomo, la adaptación a distintos ritmos de aprendizaje y la flexibilidad en el proceso de enseñanza-aprendizaje (Blasco-Serrano et al., 2018), siendo estos beneficios posibles gracias al alcance que tiene el uso adecuado de la tecnología en la educación.

Estudios recientes como el de Sánchez-Rivas et al. (2019) han demostrado que este modelo permite la optimización del tiempo invertido en el aula. Mientras que Uzunboylu y Karagözlü (2017) en una revisión bibliográfica encontraron que el FC puede ser efectivo y aplicable en muchos contextos. Además, Mcgivney-Burelle y Xue (2013) evidenciaron en una investigación que los estudiantes preferían este tipo de pedagogía, específicamente la disponibilidad de videos y el uso de tiempo de clases para la resolución de problemas, ya que obtenían mejores resultados en sus evaluaciones.

\section{Dificultades en la Enseñanza Aplicando el Flipped Classroom}

Como cualquier propuesta didáctica, si el FC no se aplica apropiadamente los resultados pueden ser desfavorables y puede causar en los estudiantes frustración y desánimo ante un posible fracaso en el proceso de aprendizaje, contrario a su objetivo. El estudio realizado por Bagley (2020) evidenció que los participantes de un aula donde el modelo fue aplicado sintieron una ausencia significativa del docente y lo consideraron un incumplimiento al contrato didáctico de crear las condiciones necesarias para la adquisición del conocimiento.

Estos estudiantes sintieron que los videos previos a la clase eran ineficaces en la preparación a la clase con el docente y que las hojas de trabajo asignadas no estaban bien estructuradas y eran muy difíciles para mediar su aprendizaje. De allí la importancia de diseñar la asignatura desde el inicio del programa pensando en la construcción del conocimiento que debe hacer el estudiante por sí solo y que luego será complementado por el docente con 
actividades que se conectan directamente con el trabajo previamente realizado.

Otra dificultad puede presentarse debido a la falta de madurez y poco grado de responsabilidad que pueden tener los alumnos de estudios medios o inclusive de los primeros niveles de pregrado, de manera que el método FC puede ser más efectivo con estudiantes de niveles superiores. Sin embargo, se debe incentivar su aplicación en niveles inferiores, ya que esto contribuiría a formar desde los primeros años de estudio estudiantes proactivos y autónomos en su propio proceso de aprendizaje (Del Arco Bravo et al., 2019).

Por otra parte, el uso de las TIC ha sido clave en la aplicación de este modelo, ya que permite y facilita la modificación de los procesos de seguimiento y evaluación en el aula; pero esto solo es posible si los profesores las dominan (Aldon y Panero, 2020). Y es que las carencias en una formación permanente del profesorado universitario en aspectos metodológicos y de tecnología educativa puede afectar la interacción entre el alumno y docente (Sánchez-Rivas et al., 2019) siendo contraproducente en el proceso de enseñanza-aprendizaje. Por ello, la importancia de instruirlos en el uso de nuevas herramientas tecnológicas antes de aplicar el FC.

\section{Experiencias del Flipped Classroom en las Clases de Matemática}

En los últimos años la aplicación del modelo pedagógico Flipped Classroom ha tenido mucho auge en la enseñanza de las matemáticas y es probable que continúe en aumento (De Araujo et al., 2017). Cevikbas y Kaiser (2020) consideran que en la actualidad el FC es un enfoque pedagógico innovador con un gran potencial para transformar la enseñanza de las matemáticas. Los resultados del estudio realizado por estos investigadores mostraron que haciendo uso de un modelo FC bien diseñado los docentes pueden desarrollar el potencial matemático de sus estudiantes.

Por su parte, De Araujo et al. (2017) realizaron un estudio sobre una maestra de matemáticas que implementó en FC haciendo uso de videos instructivos y recursos multimedia diseñados por ella como reemplazo a los textos escolares y concluyeron que los profesores de matemática deben considerar los videos utilizados en las aulas invertidas como materiales curriculares y no simplemente como representaciones de instrucciones. En concordancia, Voigt et al. (2020) mostraron en una reciente investigación la importancia de unir los videos asignados a los estudiantes con los componentes curriculares por medio de un diseño heurístico que permitía capacitar a los estudiantes para pensar críticamente sobre problemas matemáticos de forma personalizada antes de asistir a clase en un colectivo. 
Del mismo modo, otros autores consideran que, aunque no es el único recurso audiovisual útil en el modelo FC, los videos tienen un importante impacto en la comprensión de conceptos y procedimientos involucrados en la exploración del conocimiento por parte de los estudiantes (Bagley, 2020; Basso-Aránguiz et al., 2018; Matzumura-Kasano et al., 2018; Mcgivney-Burelle y Xue, 2013; Uzunboylu y Karagözlü, 2017).

Siguiendo las ideas de los autores mencionados, se ha evidenciado que el FC es un modelo que bien diseñado puede generar resultados exitosos en el desarrollo de habilidades matemáticas de los estudiantes y que un importante recurso para su implementación son los videos. No obstante, estos videos deben ser diseñados pensando en la autoformación crítica y reflexiva del estudiante al momento de visualizarlos. Así, fundamentados en lo anterior, esta investigación utilizó como principal recurso audiovisual en la aplicación del modelo FC videos interactivos creados en la plataforma H5P que viene incorporada en la versión 3.9 de Moodle y con la cual contaron todos los participantes de la investigación.

\section{Recursos Tecnológicos Utilizados para Asistir el Modelo Flipped Classroom}

Como se ha mencionado antes, la tecnología juega un papel fundamental en la implementación del FC. En consonancia, esta investigación acudió fundamentalmente al uso tres herramientas tecnológicas: Moodle, H5P y Zoom. Moodle, por su acrónimo del inglés Modular Object Oriented Dynamic Learning Environment, es una herramienta de software libre con un ambiente de trabajo fácil de usar y es uno de los sistemas de gestión de aprendizaje más utilizados por las universidades a nivel mundial (González-Segura et al., 2018; Moreno y Montoya, 2015; Tapia-León et al., 2015). Además, es flexible y promueve los procesos formativos y de trabajo colaborativo en medio una población estudiantil que es digitalmente nativa (Río et al., 2018). Esta plataforma ayuda a la implementación del FC al propiciar un ambiente flexible siendo este uno de los pilares fundamentales del método.

H5P (https://h5p.org) es una plataforma de código abierto que permite crear recursos virtuales de aprendizajes interactivos basados en un lenguaje HTML5 (Rossetti et al., 2020). Esta herramienta proporciona una solución innovadora en el ámbito tecnológico-educativo permitiendo al docente generar nuevos recursos interactivos de aprendizaje (Rekhari y Sinnayah, 2018) que brinden oportunidades a los estudiantes de pensar críticamente sobre lo que aprenden (Sinnayah et al., 2021). Específicamente, con este recurso se pueden diseñar videos interactivos que permiten al participante interactuar con el contenido del video 
respondiendo a diferentes estilos de preguntas que son insertadas en el video.

Zoom (https://zoom.us) es una plataforma privada que ofrece un servicio de videoconferencias para programar reuniones virtuales en tiempo real con otras personas. La pandemia causada por el COVID-19 ha impulsado exponencialmente el uso de este servicio en todas las industrias y en particular en la educación. Con ayuda de esta herramienta muchas instituciones educativas han logrado realizar virtualmente todas las reuniones que en situaciones normales hacían de manera presencial, especialmente los encuentros entre profesores y estudiantes se han podido hacer sincrónicamente con ayuda de Zoom. La herramienta permitió romper la barrera de la no-presencialidad para desarrollar los momentos donde el estudiante necesita de una comunicación instantánea con su tutor.

\section{El Método}

Este estudio se enmarcó bajo un enfoque cualitativo y con un diseño de investigaciónacción (Hernández et al., 2014) que buscó propiciar un cambio en una realidad educativa inmersa en medio de la tecnología. Su objetivo fue determinar la eficacia del modelo Flipped Classroom aplicado en las clases virtuales de matemática sobre la participación de los estudiantes en sus procesos de aprendizaje. Para ello, se utilizó una muestra conformada por 133 estudiantes del primer nivel de ingeniería de la Universidad Politécnica Salesiana del Ecuador durante el período 2020-2021 y cuyas edades comprendían entre los 18 y 21 años; la muestra estuvo repartida en cuatro cursos que estuvieron a cargo del investigador, dos de álgebra lineal y dos de cálculo diferencial.

Las clases fueron orientadas de manera virtual, apoyadas principalmente por la plataforma Zoom para las clases sincrónicas con el docente (aprendizaje asistido) y un ambiente virtual de aprendizaje cooperativo (AVAC) diseñado con el sistema de gestión de aprendizaje Moodle v3.9 para el trabajo autónomo del estudiante. La investigación se llevó a cabo en cuatro fases.

\section{Fases de la Investigación Fase 1. Planificación de la asignatura}

Esta fase se ejecutó previo al inicio del semestre y finalizó en la primera sesión de clases. En primer lugar, se diseñaron los sílabos de las asignaturas considerándose la distribución de horas para las actividades que fueron divididas en dos grupos: trabajo autónomo 
y clases sincrónicas (aprendizaje asistido). Adicional, se preparó un material audiovisual en PowerPoint para presentar a los estudiantes en la primera sesión de clases el método de enseñanza Flipped Classroom que se utilizaría a lo largo del semestre; el propósito de esta sesión fue fomentar desde el inicio la cultura del aprendizaje basado en un modelo educativo centrado en el estudiante.

\section{Fase 2. Diseño de recursos didácticos}

Esta fase se llevó a cabo durante todo el semestre, consistió en seleccionar un video de YouTube para cada clase, el mismo debía cumplir algunas condiciones: ajustarse al tema de la clase, ser conciso y preciso, tener una duración máxima de 15 minutos y tener calidad audiovisual. Una vez seleccionado el video de la clase, fue utilizado para diseñar un recurso H5P (video interactivo) en el AVAC. El diseño del video interactivo permitió al estudiante responder durante su reproducción preguntas que le fueron incrustadas (de opción múltiple, verdadero y falso, complementación, apareamiento, entre otras). Cuando el estudiante llegaba a la pregunta el video se detenía, esto con el fin de darle el tiempo de analizar y responderla para continuar.

Además, con la intención de captar la atención del estudiante en todo momento, el video interactivo no permitía retroceder ni adelantar su reproducción, pero sí pausarla. En algunos casos, cuando el docente lo consideró necesario, también se incrustaron comentarios para hacer alguna aclaratoria del tema. El estudiante debía resolver esta actividad antes de cada clase en un tiempo recomendado de máximo 30 minutos. En este momento el aula fue invertida, el papel protagónico en el aprendizaje lo tomó el estudiante, pues guiado con el recurso H5P el único responsable de su aprendizaje fue él.

\section{Fase 3. Secuencia didáctica para las clases}

Esta fase fue pensada para llevarse a cabo en torno a cada clase desarrollada en tres momentos: i) Revisión del video interactivo (trabajo autónomo), éste sirve como una introducción a la clase y garantiza los conocimientos previos del estudiante, la actividad puede ser evaluada asignándole una ponderación en el AVAC como puntos extras para motivarlos o bien como parte de la evaluación sumativa, en este estudio se consideró como puntos extras; ii) Presentación de la clase sincrónica (aprendizaje asistido), por medio de la plataforma Zoom 
el docente desarrolla el tema a tratar partiendo del aprendizaje adquirido por los estudiantes en los videos interactivos, en este punto el alumno debe tener una base para iniciar con el estudio del tema, y el profesor puede dedicarse a profundizar los aspectos más relevantes y/o que requieran de mayor atención; iii) Resolución de una actividad en el AVAC (trabajo autónomo), una vez finalizada la clase sincrónica es importante que el estudiante consolide sus conocimientos con la práctica, para ello se asignaron actividades de repaso enfocadas sobre el tema tratado en la clase. Moodle tiene varias actividades que pueden ser útiles en esta etapa: cuestionarios, talleres, foros, tareas, ...). En las asignaturas donde se desarrolló esta investigación se utilizaron como actividades el cuestionario (con un amplio banco de preguntas elaboradas previamente) y las tareas (asignadas por grupos de trabajo).

Es importante mencionar que toda la secuencia didáctica quedó registrada en el AVAC, esto permitió al docente reasignar actividades a los participantes en caso de considerarlo necesario para que éstos obtuvieran el logro de aprendizaje esperado. Del mismo modo, el estudiante tuvo la libertad de revisar los videos interactivos y las grabaciones de las clases sincrónicas las veces que lo consideró necesario.

\section{Fase 4. Evaluación del proceso de formación}

Gracias a la facilidad que presta Moodle en la corrección individualizada de las actividades de cada participante; la última fase de la secuencia didáctica (resolución de una actividad en el AVAC), permitió al docente evaluar de manera continua y sistemática el avance en la adquisición de los logros de aprendizaje del estudiante. Así pues, al finalizar el semestre el estudiante (orientado por el docente) pudo tomar a tiempo las acciones necesarias para mejorar su rendimiento académico, la intención es considerar la evaluación como parte del proceso de formación y como tal debe atenderse durante el desarrollo del semestre y no finalizado éste.

Durante todo el proceso formativo es el estudiante quien tiene el papel protagónico y el docente solo actúa como un mediador en el proceso de aprendizaje. Estas cuatro fases pueden resumirse en la figura 1. 


\section{Figura 1}

Fases de la investigación

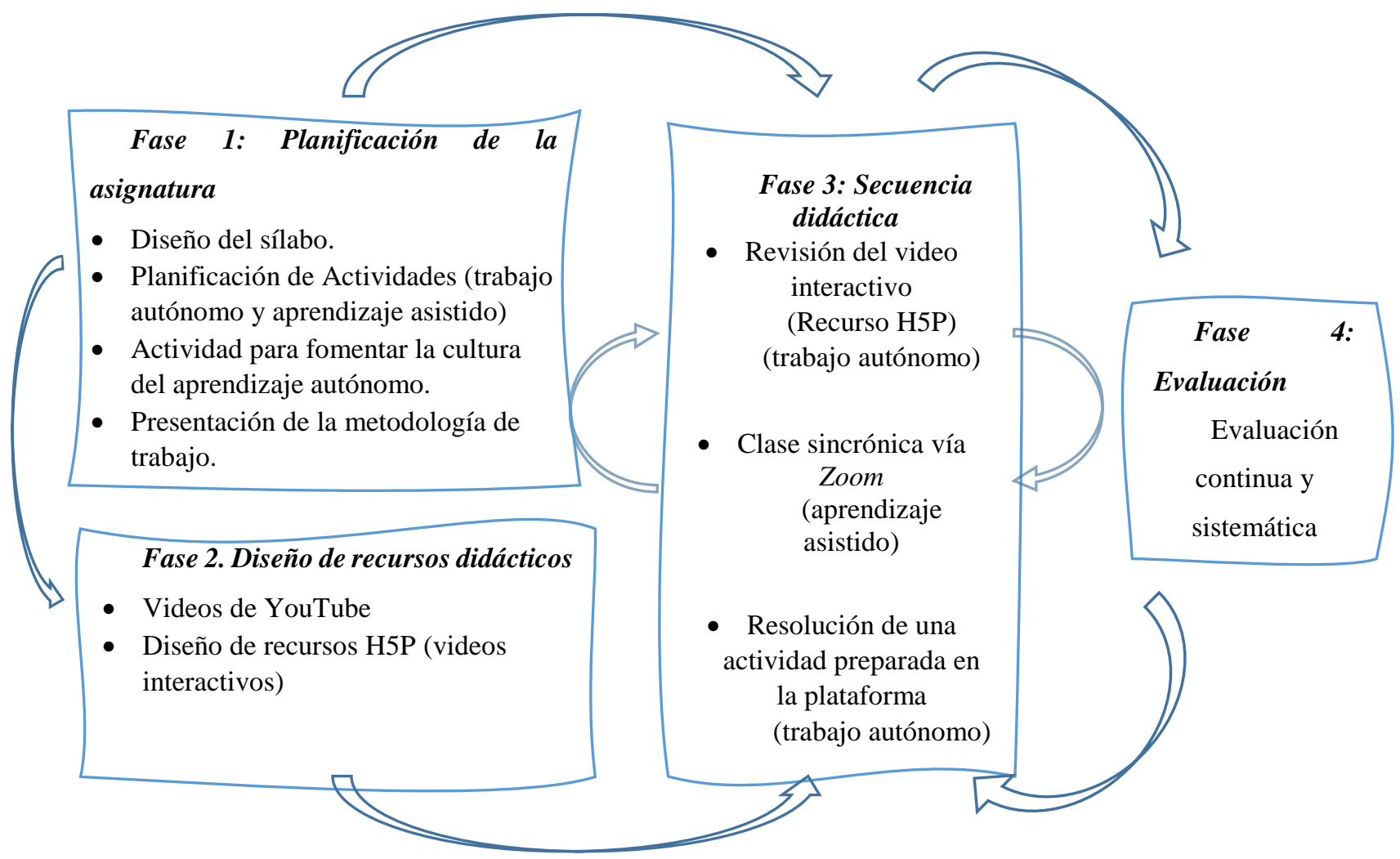

Nota: La evaluación es un proceso que se lleva a cabo durante toda la secuencia didáctica.

\section{Recolección de la Información}

Para la recolección de la información se aplicó la observación durante todo el semestre por parte del investigador y una encuesta semiestructurada diseñada en el AVAC para determinar el grado de satisfacción que los estudiantes tenían respecto al método de invertir el aula como estrategia de enseñanza. La encuesta estuvo conformada por los siguientes ocho (8) cuestionamientos previamente revisados y validados por cinco expertos en el tema.

1. ¿Revisar videos interactivos en la plataforma le permitieron tener una mejor comprensión de los temas al momento de ver las clases sincrónicas con el profesor? ¿Por qué?

2. ¿Revisar un video interactivo en la plataforma es más útil para su proceso de aprendizaje que ver el video directamente en YouTube? ¿Por qué? 
3. ¿Responder a las preguntas que se presentaban durante la reproducción de los videos le ayudó a reflexionar sobre el tema tratado? ¿Por qué?

4. ¿La duración de los videos (de 5 a 15 minutos) le pareció que fue suficiente para comprender el tema tratado? ¿Por qué?

5. ¿Las clases sincrónicas con el profesor le fueron de ayuda para complementar los contenidos tratados en los videos interactivos? ¿Por qué?

6. ¿Las actividades de repaso (propuesta para después de la clase sincrónica) le ayudaron a consolidar el conocimiento de los temas tratados en clase?

7. ¿Durante el desarrollo del semestre sintió que la responsabilidad de su aprendizaje dependía más de usted que de su profesor?

8. ¿Siente que durante el semestre tuvo un papel protagónico en su proceso de aprendizaje? ¿Por qué?

Con base en estos cuestionamientos se realizó una categorización (cuadro 1) que apoyada en la observación permitiera al investigador clasificar las respuestas de los estudiantes para analizar la variable de investigación definida por la eficacia del modelo FC sobre la participación del alumnado en sus procesos de aprendizaje.

\section{Cuadro 1}

Categorización de la variable de investigación

\begin{tabular}{cc}
\hline Variable de investigación & \multicolumn{1}{c}{ Categorías } \\
\hline & - Utilidad de los videos interactivos para el \\
& aprendizaje. \\
Eficacia del modelo flipped classroom aplicado & - Duración de los videos interactivos. \\
en las clases virtuales de matemática sobre la & - Complementación del tema con las clases \\
participación de los estudiantes en sus procesos & sincrónicas. \\
de aprendizaje & Complementación del tema con las actividades \\
& de repaso. \\
& Participación protagónica del estudiante en su \\
& proceso de aprendizaje. \\
\hline
\end{tabular}

Nota: Siguiendo los lineamientos de una investigación de carácter cualitativo, el objetivo del análisis no ha sido el establecimiento de relaciones causa-efecto, sino la identificación de patrones de respuestas que permitan determinar la eficacia del modelo $F C$ en las clases virtuales de matemáticas.

En el cuadro 2, se muestra una síntesis de la metodología aplica en esta investigación: 


\section{Cuadro 2}

Síntesis de la metodología aplicada

\begin{tabular}{cccc}
\hline \multicolumn{1}{c}{ Enfoque } & \multicolumn{1}{c}{ Diseño } & \multicolumn{1}{c}{ Técnicas } & Forma de análisis \\
\hline \multirow{2}{*}{ Cualitativo } & Investigación - Acción & Observación & $\begin{array}{l}\text { Análisis de } \\
\text { observación }\end{array}$ \\
\cline { 3 - 4 } & & $\begin{array}{l}\text { Encuesta } \\
\text { semiestructurada }\end{array}$ & Análisis de contenido
\end{tabular}

\section{Análisis y Discusión de los Resultados}

Para realizar el análisis de los resultados de esta investigación se consideraron las 5 categorías mencionadas basadas en la observación del investigador sobre los grupos de estudiantes y sus correspondientes respuestas en la encuesta aplicada.

\section{Utilidad de los videos interactivos para el aprendizaje.}

Desde la mirada del docente, en la introducción a las clases sincrónicas por medio de la plataforma Zoom pudo apreciarse una participación considerable de los estudiantes lo que evidenciaba que conocían del tema que se iba a tratar; es decir, era notorio que los alumnos traían conocimientos previos a la clase. Sin embargo, en muchos casos no se apreciaba una formalidad en los conceptos matemáticos que exponían, obligando al docente a retomar estos conceptos con mayor atención antes de continuar con la clase.

Desde la mirada del estudiante, las respuestas a la encuesta evidenciaron un alto grado de aceptación en la asignación de videos interactivos como material de apoyo disponible previo a cada clase, ya que consideraban les daba un fundamento teórico antes de recibirla. Cabe destacar la importancia que se dio a las preguntas realizadas durante la reproducción de los videos, siendo éstas un elemento característico en la participación del estudiante en la construcción de su conocimiento, ya que se veían obligados a analizar los conceptos presentados y en última instancia revisar la bibliografía correspondiente. Esto apoya las ideas de Voigt et al. (2020) al mencionar la importancia de unir los videos con los componentes curriculares por medio de un diseño heurístico que fomente un pensamiento crítico en los estudiantes.

“Es mucho más útil porque el profesor nos hace una serie de preguntas durante el video interactivo lo cual nos permite relacionarnos más con el tema, si lo 
viéramos directamente en YouTube no tuviéramos acceso a las preguntas que son de aspectos muy esenciales y a su vez importantes"

“Sí, porque además de ver un video corto pero conciso, existían preguntas y eso hacía más fácil la comprensión de la clase”

"Sí, aunque debo admitir que en algunas preguntas me tomaba mucho tiempo responder porque tenía que revisar materia o analizar de una manera más profunda."

Algunos estudiantes hicieron referencia a la necesidad de poder retroceder el video porque consideraban que se reproducía muy rápido y no captaban las ideas que se trataban de enseñar. Sin embargo, esta opción fue deshabilitada pensando en forzar al estudiante a estar atento al tema, no obstante, el video podía repetirse las veces que fueran necesarias para el alumno, pero con la desventaja para él, de tener que verlo completo de nuevo.

"Creo que en los videos es necesario poder retroceder un poco ya que en ocasiones no se puede captar a la primera la idea que se nos trata de dar o no se comprende bien alguna parte, por lo cual considero que sería bueno poder retroceder un poco el video en vez de tener que ver todo de nuevo"

\section{Duración de los videos interactivos.}

Un aspecto importante a considerar en el diseño de los videos fue su tiempo de duración. Por ejemplo, las clases sincrónicas tenían una duración aproximada de 45 a 60 minutos y durante ese tiempo el docente podía notar cansancio y aburrimiento en sus alumnos, lo cual debía contrarrestar con comentarios o actividades que atrajeran de nuevo la atención de ellos. Sin embargo, en un video no es posible esta práctica, esto significa que difícilmente se podría mantener la atención de un estudiante sobre un video educativo durante un tiempo muy prolongado. Al respecto, la totalidad de los participantes estuvieron de acuerdo con que el tiempo de duración de los videos (de 5 a 15 minutos) era adecuado, ya que les generaba a lo sumo una inversión de 30 minutos para realizar la actividad; pese a que algunos decidían invertir una mayor cantidad de tiempo de acuerdo con sus necesidades.

\section{Complementación del tema con las clases sincrónicas}

Los videos interactivos solo representaban una introducción al tema en cuestión, pero 
como se mencionó antes muchos de los estudiantes llegaban a la clase con unas ideas no muy claras de los conceptos tratados. Por lo cual siempre era necesario retomar los conceptos básicos mencionados en el video para aclararlos y analizarlos en mayor detalle lo cual requería de tiempo antes de iniciar con la clase programada; esto se contrapone a los resultados de Sánchez-Rivas et al. (2019) que concluyeron en su investigación que este modelo optimizaba el tiempo en el aula. Además, considerando que las asignaturas (cálculo diferencial y álgebra lineal) eran prácticas, fue casi unísono la necesidad de resolver problemas durante las clases sincrónicas de manera que se pudieran plantear y aclarar detalladamente las dudas que se iban presentando.

"Sí, los videos son solo una pequeña ayuda para ver sobre lo qué se va a tratar, pero el profesor es el complemento porque si se presentara una duda él nos ayudaría aclarándola”

"Sí, porque el profesor explicaba más detalladamente y creo que todos entendíamos de mejor manera"

En este sentido, pudo notarse que al momento de invertir el aula siguiendo esta secuencia didáctica los videos no sustituyeron la instrucción del docente y sus clases sincrónicas (aprendizaje asistido) bajo ningún motivo podían ser omitidas, pues fueron fundamentales en el aprendizaje del estudiante. Esto coincide con las ideas de Bagley (2020) quien asegura que el docente debe crear las condiciones necesarias para la adquisición del conocimiento y que su ausencia significaría un incumplimiento al contrato didáctico.

\section{Complementación del tema con las actividades de repaso.}

El tercer momento de la secuencia didáctica Resolución de una actividad en el AVAC estaba enmarcada en el trabajo autónomo del estudiante, las actividades que eran asignadas después de la clase sincrónica fueron analizadas por el docente, aunque el AVAC ya las había calificado automática e inmediatamente para retroalimentar al estudiante. Allí, pudo notarse que gran parte de estas actividades presentaban errores en la mayoría de las respuestas dadas, sin embargo, una vez que se aplicaba una evaluación los resultados eran diferentes y favorables. En contraposición a los resultados de Bagley (2020) y según las impresiones de estos estudiantes; realizar una actividad de repaso no solo les ayudaba a complementar el tema y revisar sus falencias, sino también a familiarizarse con la plataforma en cuanto a evaluaciones se refiere y además las consideraron oportunas y acordes a los temas tratados. 
"Esas actividades nos han ayudado a reforzar nuestros conocimientos, saber en qué parte tenemos fallas para poder prepararnos más y así tener mejor calificación en las actividades calificadas"

"Dichas actividades eran de gran ayuda porque ya teníamos una idea de cómo iban a ser las preguntas de algún deber o examen y repasamos ahí”

“Claro que sí, así si nos equivocamos podemos analizar el error y no cometer lo mismo en el examen, preferible equivocarme en todo en el de repaso que en el examen"

Así pues, es importante considerar en el método FC la evaluación como un proceso continuo de formación, que no solo deben ser vistas como una asignación numérica para representar un logro de aprendizaje sino también como una mirada al desarrollo de las habilidades donde necesariamente existirán errores en el camino.

\section{Participación protagónica del estudiante en su proceso de aprendizaje}

Habiendo llevado a cabo la secuencia didáctica fundamentada en el modelo Flipped Classroom los alumnos participantes del estudio manifestaron sentir un papel protagónico en su proceso de aprendizaje. Un aspecto mencionado hizo referencia a que la matemática se aprende practicándose y a tal fin el docente debe establecer actividades que el estudiante debe resolver autónomamente con las herramientas para consultar e instruirse de manera sistemática y orientada.

Con este estudio se demostró que los videos interactivos y las actividades de repaso luego de las clases sincrónicas permiten lograr este propósito en las clases virtuales de matemática; coincidiendo con los resultados de Uzunboylu y Karagözlü (2017) quienes en una revisión bibliográfica encontraron que el método FC puede ser efectivo y aplicable en muchos contextos. Esta investigación además refuerza las ideas de Cevikbas y Kaiser (2020) quienes aseguran que este enfoque pedagógico e innovador FC cuenta con un gran potencial para transformar la enseñanza de las matemáticas.

\section{Conclusiones}

Respecto a la pregunta de investigación formulada: ¿Cómo el modelo Flipped Classroom usado en las clases virtuales de matemática puede aumentar la participación del 
estudiante en su proceso de aprendizaje?, puede mencionarse lo siguiente:

Los estudiantes que participaron en el estudio tuvieron un alto grado de aceptación con la asignación de videos interactivos previo a las clases sincrónicas. Sin embargo, a pesar de llegar a éstas con conocimientos previos del tema en cuestión que les facilitaba una participación activa en el desarrollo de la clase, en muchos de los casos se carecía de formalidad de los conceptos matemáticos planteados en el video; lo cual implicaba un tiempo extra en la clase del profesor inhibiendo la optimización del tiempo que según la teoría debía generar la aplicación del modelo Flipped Classroom.

Por otro lado, los videos con preguntas y comentarios incrustados en ellos que fueron diseñados en la plataforma H5P, jugaron un papel fundamental en la participación de la construcción del conocimiento del estudiante; al obligarlo a cuestionarse continuamente sobre el tema mientras reproducía el video, lo cual permite resaltar la importancia de utilizar en el modelo FC más que videos, videos interactivos.

Las clases sincrónicas que representaron un aprendizaje asistido fueron necesarias en todo momento del proceso de aprendizaje, los estudiantes necesitan de un guía al momento de construir y asimilar los conceptos matemáticos, si bien los videos fueron un importante recurso nunca sustituyeron al docente.

Las matemáticas necesitan de práctica para ser aprendidas, no se aprende viendo, se aprende haciendo. En este sentido, las actividades de prácticas asignadas luego de las clases sincrónicas también contribuyeron en gran medida a la participación del estudiante en la construcción de su conocimiento. En conclusión, el modelo Flipped Classroom aplicado en las clases virtuales de matemática con videos interactivos y actividades de repaso con retroalimentación inmediata contribuye a la participación de los estudiantes en sus procesos de aprendizajes, aunque no optimiza el tiempo en las clases sincrónicas con el profesor.

\section{Referencias}

Aldon, G., y Panero, M. (2020). Can digital technology change the way mathematics skills are assessed? ZDM Mathematics Education, 52(7), 1333-1348. https://doi.org/10.1007/s11858-020-01172-8 
Amaya, A., Cantú, D., y Marreros, J. (2021). Análisis de las competencias didácticas virtuales en la impartición de clases universitarias en línea, durante contingencia del COVID-19. RED. Revista de Educación a Distancia, 21(65). http://dx.doi.org/10.6018/red.426371 Bagley, S. (2020). The Flipped Classroom, Lethal Mutations, and the Didactical Contract : A $\begin{array}{llll}\text { Cautionary } & \text { Tale. } & \text { PRIMUS, } & \text { 243-260. }\end{array}$ https://doi.org/10.1080/10511970.2018.1555196

Basso-Aránguiz, M., Bravo-Molina, M., Castro-Riquelme, A., y Moraga-Contreras, C. (2018). Propuesta de modelo tecnológico para Flipped Classroom (T-FliC) en educación superior. Revista Electrónica Educare, 22(2), 1. https://doi.org/10.15359/ree.22-2.2

Blasco-Serrano, A. C., Lorenzo, J., y Sarsa, J. (2018). Percepción de los estudiantes al 'invertir la clase' mediante el uso de redes sociales y sistemas de respuesta inmediata. Revista de Educación a Distancia (RED), 57(6), 1-19. https://doi.org/10.6018/red/57/6

Blasco-Serrano, A. C., Lorenzo, J., y Sarsa, J. (2016). The flipped classroom and the use of educational software videos in initial teaching education. Qualitative study. @ Tic. Revista D'Innovació Educativa, O(17). https://doi.org/10.7203/attic.17.9027

Borba, M. C., Askar, P., Engelbrecht, J., Gadanidis, G., Llinares, S., y Aguilar, M. S. (2016). Blended learning, e-learning and mobile learning in mathematics education. ZDM, 48(5), 589-610. https://doi.org/10.1007/s11858-016-0798-4

Castellanos Sánchez, A., Sánchez Romero, C., y Calderero Hernández, J. F. (2017). Nuevos modelos tecnopedagógicos. Competencia digital de los alumnos universitarios. Revista Electrónica de Investigación Educativa, 19(1), 1-9. https://doi.org/10.24320/redie.2017.19.1.1148

CEPAL-UNESCO. (2020). La educación en tiempos de la pandemia de COVID-19. Geopolitica, 11. https://repositorio.cepal.org/handle/11362/45904

Cevikbas, M., y Kaiser, G. (2020). Flipped classroom as a reform-oriented approach to teaching mathematics. ZDM Mathematics Education, 52(7), 1291-1305. https://doi.org/10.1007/s11858-020-01191-5

De Araujo, Z., Otten, S., y Birisci, S. (2017). Teacher-created videos in a flipped mathematics class: digital curriculum materials or lesson enactments? ZDM, 49(5), 687-699. https://doi.org/10.1007/s11858-017-0872-6

Del Arco Bravo, I., Flores Alarcia, Ó., y Silva García, P. (2019). El desarrollo del modelo flipped classroom en la universidad: impacto de su implementación desde la voz del 
estudiantado. Revista de Investigación Educativa, 37(2), 451-469. https://doi.org/10.6018/rie.37.2.327831

Engelbrecht, J., Borba, M. C., Llinares, S., y Kaiser, G. (2020). Will 2020 be remembered as the year in which education was changed? ZDM - Mathematics Education, 52(5), 821824. https://doi.org/10.1007/s11858-020-01185-3

Flipped Learning Network (FLN) (2014). What Is Flipped Learning? https://flippedlearning.org/wp-content/uploads/2016/07/FLIP_handout_FNL_Web.pdf

González-Segura, C. M., García-García, M., y Menéndez, V. H. (2018). Análisis de la evaluación de competencias y su aplicación en un sistema de gestión del aprendizaje. Un caso de studio. Revista de Educación a Distancia (RED), 58. https://doi.org/10.6018/red/58/3

Hernández, R., Fernández, C., y Baptista, M. del P. (2014). Metodología de la investigación. (6ta. Ed.). Mc Graw Hill Education.

Latorre-Cosculluela, C., Vázquez-Toledo, S., Rodríguez-Martínez, A., y Liesa-Orús, M. (2020). Design Thinking: creatividad y pensamiento crítico en la universidad. Revista Electrónica de Investigación Educativa, 22, 1-13. https://doi.org/10.24320/redie.2020.22.e28.2917

Lovón, M., y Cisneros, S. (2020). Repercusiones de las clases virtuales en los estudiantes universitarios en el contexto de la cuarentena por COVID- 19: El caso de la PUCP. Porpósitos y Repreresentaciones. Revista de Psicología Educativa, 8(3). https://doi.org/https://dx.doi.org/10.20511/pyr2020.v8nSPE3.588

Lucas, C., y Gascón, J. (2019). Las tres dimensiones del problema didáctico del cálculo diferencial elemental. Avances de Investigación En Educación Matemática, 16, 40-56. https://doi.org/10.35763/aiem.v0i16.277

Martínez-Olvera, W., y Esquivel-Gámez, I. (2018). Uso del modelo de aprendizaje invertido en un bachillerato público. Revista de Educación a Distancia (RED), 58. https://doi.org/10.6018/red/58/11

Matzumura-Kasano, J. P., Gutiérrez-Crespo, H., Zamudio-Eslava, L. A., y Zavala-Gonzales, J. C. (2018). Flipped Learning Model to Achieve Learning Goals in the Research Methodology Course in Undergraduate Students. Revista Electrónica Educare, 22(3), 1-21. https://doi.org/10.15359/ree.22-3.9

Mcgivney-Burelle, J., y Xue, F. (2013). Flipping Calculus. PRIMUS, 23(5), 477-486. https://doi.org/https://doi.org/10.1080/10511970.2012.757571 
Moreno, J., y Montoya, L. F. (2015). Use of a gamified virtual learning environment as didactic strategy in a pre-calculus course: Case study in the National University of Colombia. RISTI - Revista Iberica de Sistemas e Tecnologias de Informacao, 16, 1-16. https://doi.org/10.17013/risti.16.1-16

Ndlovu, M., Ramdhany, V., Spangenberg, E. D., y Govender, R. (2020). Preservice teachers' beliefs and intentions about integrating mathematics teaching and learning ICTs in their classrooms. ZDM Mathematics Education, 52(7), 1365-1380. https://doi.org/10.1007/s11858-020-01186-2

Ramirez, A. (2020). El uso de la tecnología en el aula para motivar a la participación en clases y evaluar sus conocimientos y grado de comprensión. In F. Knop (Ed.), Escritos en la Facultad. Reflexión Pedagógica. Edición VIII Ensayos de estudiantes de la Facultad de Diseño y Comunicación (1ra Edición, pp. 210-211). Universidad de Palermo. https://fido.palermo.edu/servicios_dyc/publicacionesdc/archivos/855_libro.pdf\#page= $\underline{210}$

Rekhari, S., y Sinnayah, P. (2018). Research and development in higher education: [ Re ] valuing higher education $\mathrm{h} 5 \mathrm{p}$ and innovation in anatomy and physiology teaching. HERDSA Annual International Conference, 41, 191-205. www.herdsa.org.au

Río, C. J., Calle, R. C., Elena Martín Pastor, M., y Robaina, N. F. (2018). Rendimiento académico en educación superior y su asociación con la participación activa en la plataforma Moodle. Estudios Sobre Educación, 34, 177-198. https://doi.org/10.15581/004.34.177-198

Rossetti, S. R., García Ramirez, M. T., Rojas Rodriguez, I. S., Morita Alexander, A., y Coronado García, M. A. (2020). Objeto virtual de aprendizaje creado con plataforma de software libre H5P y su impacto en el aprendizaje. Revista Cubana de Ciencias Informáticas, 14(2), 1-14.

Sánchez-Rivas, E., Sánchez-Rodríguez, J., y Ruiz-Palmero, J. (2019). Percepción del alumnado universitario respecto al modelo pedagógico de clase invertida. Magis, Revista Internacional de Investigación En Educación, 11(23), 151-168. https://doi.org/10.11144/Javeriana.m11-23.paur

Sinnayah, P., Salcedo, A., y Rekhari, S. (2021). Reimagining physiology education with interactive content developed in H5P. Advances in Physiology Education, 45(1), 7176. https://doi.org/10.1152/advan.00021.2020 
Tapia-León, M., Peñaherrera-Larenas, F., y Cedillo-Fajardo, M. (2015). Comparación de los LMS Moodle y CourseSites de Blackboard usando el modelo de aceptación tecnológica $\begin{array}{llll}\text { TAM. Revista } & \text { Ciencia }\end{array}$ https://doi.org/https://doi.org/10.29076/issn.2528-7737vol8iss16.2015pp78-85p

Trouche, L., Rocha, K., Gueudet, G., y Pepin, B. (2020). Transition to digital resources as a critical process in teachers' trajectories: the case of Anna's documentation work. ZDM Mathematics Education, 52(7), 1243-1257. https://doi.org/10.1007/s11858-020$\underline{01164-8}$

Uzunboylu, H., y Karagözlü, D. (2017). The Emerging Trend of the Flipped Classroom: A Content Analysis of Published Articles between 2010 and 2015. Revista de Educación a Distancia (RED), 54. https://doi.org/10.6018/red/54/4

Vahey, P., Kim, H.-J., Jackiw, N., Sela, H., y Knudsen, J. (2020). From the static to the dynamic: teachers' varying use of digital technology to support conceptual learning in a curricular activity system. ZDM Mathematics Education, 52(7), 1275-1290. https://doi.org/10.1007/s11858-020-01182-6

Voigt, M., Fredriksen, H., y Rasmussen, C. (2020). Leveraging the design heuristics of realistic mathematics education and culturally responsive pedagogy to create a richer flipped classroom calculus curriculum. ZDM Mathematics Education, 52(5), 1051-1062. https://doi.org/10.1007/s11858-019-01124-X 